\title{
Viscoelastic and rheological properties of carboxymethyl cellulose /starch/graphite oxide as superabsorbent hydrogel nano composites (SHNCs)
}

\author{
Auda Jabbar Braihi ${ }^{1}$, Sihama Issa Salih ${ }^{2}$, Fadhel Abbas Hashem ${ }^{2}$ \\ ${ }^{1}$ Babylon University, College of Materials Engineering, Polymer and Petrochemical Industries Department, Babylon, Iraq \\ ${ }^{2}$ University of Technology, Materials Engineering, Baghdad, Iraq
}

Email address:

auda_1964@yahoo.com (A. J. Braihi), sihama_salih@yahoo.com (S. I. Salih), hashembsb@yahoo.com (F. A. Hashem)

\section{To cite this article:}

Auda Jabbar Braihi, Sihama Issa Salih, Fadhel Abbas Hashem. Viscoelastic and Rheological Properties of Carboxymethyl Cellulose /Starch/Graphite Oxide as Superabsorbent Hydrogel Nano Composites (SHNCs). International Journal of Materials Science and Applications. Special Issue: Steel and Direct Reduced Iron (Sponge Iron) Industry. Vol. 4, No. 2-1, 2015, pp. 30-36.

doi: 10.11648/j.ijmsa.s.2015040201.16

\begin{abstract}
Uncross-linked carboxymethyl cellulose (CMC) / starch blend used to prepare two hydrogels; the first is crosslinked CMC / starch with aluminum sulfate octadecahydrate cross-linker and the second is SHNCs manufactured from incorporation of $0.3 \mathrm{wt} \%$ nano graphite oxide (GO) in the above cross-linked blend. Viscoelastic and rheological properties of these two hydrogels were studied and compared with the uncross-linked blend. Results showed that the cross-linking process increases the blend miscibility and converted it to miscible blend. The SHNCs hydrogel has the higher $\mathrm{T}_{\mathrm{g}}$ and the uncrosslinked blend has the higher Tan $\delta$. GO addition causes increment in some viscoelastic parameters such as $G^{*}$, G', and $G^{\prime \prime}$, and decrement in damping parameters and make the damping behavior more stable at lower frequencies, and causes increment for all rheological parameters $\left(\eta^{*}, \eta_{\mathrm{o}}, \eta_{\mathrm{f}}\right.$, and $\left.\mathrm{G}_{\mathrm{N}}{ }^{\circ}\right)$. GO addition leads, also, to the absence of the shear thickening behavior and increases both the entanglement density and the molecular weight average. Both hydrogels exhibited broad molecular weight distribution (MWD) and highly cross-linking degree and their elastic behavior predominates; makes them rigid specially at higher frequencies.
\end{abstract}

Keywords: Viscoelastic Properties, Rheology, Superabsorbent Hydrogel Nanocomposites (SHNCs), Graphite Oxide(GO), Carboxymethyl Cellulose(CMC)

\section{Introduction}

Polymers composed of long molecular chains have unique viscoelastic properties, which combine the characteristics of elastic solids and Newtonain fluids [1].

Viscoelasticity makes the material's response to stressstrain behavior time dependent and their deformation partially reversible. Polymer melt considers as a nonNewtonian fluid. This behavior can be attributed to its molecular structure. Polymers consist of long molecules that entangle with each other, forming several flexible, reversible "joints". These enable different conformations of the molecules by a rotation along the backbone and cause the elastic behavior of polymer melts. The chains can also move with respect to each other by a crawling kind of movement called reptation. These rotation and reptation occurring above the glass transition temperature of the polymer are called
Brownian motions, and they tend to return the molecules towards the equilibrium, i.e., to the energetically most preferable state, after being oriented by applying deforming stress. This will not however, occur immediately after removing the stress within a certain relaxation time, dependent on the molecular characteristics of the polymer [2].

In the dynamic mechanical thermal analysis (DMTA), the mechanical response of a viscoelastic material is studied as a function of temperature and time, while it is subjected to a sinusoidal strain[3]. For viscoelastic materials, DMTA and rheological tests can be used to:

1 - Determine the viscoelastic parameters $\left(\mathrm{G}^{*}, \mathrm{G}^{\prime}, \mathrm{G}^{\prime \prime}, \tan \delta\right.$, $\eta^{*}, \eta_{\mathrm{o}}$ ) as a function of temperature, frequency or time. $\mathrm{G}^{*}$ is the complex modulus reflects the contribution of both elastic and viscous components to the material's stiffness (eqn.1) 
and measures the material's stiffness (its ability to resist deformation).

$$
\mathrm{G}^{*}=\mathrm{G}^{\prime}+\mathrm{G}^{\prime \prime}[4]
$$

$\mathrm{G}^{\prime}$ is the elastic or storage modulus, $\mathrm{G}^{\prime \prime}$ is the viscous or loss modulus, which measures the energy dissipated as heat and $\tan \delta$ is the damping coefficient or loss tangent, which is the ratio of loss and storage moduli $\left(\mathrm{G}^{\prime} / \mathrm{G}^{\prime \prime}=\tan \delta\right)$ and measures the internal friction [2]. $\delta$ is a phase angle between stress and strain(a measure of viscous response).

Complex viscosity $\left(\eta^{*}\right)$ measures the material's overall resistance to flow as a function of shear rate. In the range of low shear rates, the viscosity of a polymer solution is independent of the shear rate. This viscosity is called the zero-shear viscosity, $\eta_{0}$. [5].

2-Give information about shear-thinning and shear thickening behaviors: Shear-thinning occurs in the form of reduction in viscosity as the shear stress increases [6].This reduction is due to molecular alignments and disentanglements of the long polymer chains. Shear thickening occurs due to the increasing the viscosity with the deformation rate. Therefore shear thinning indicates rigid systems while shear thickening reflects flexible and dilute chains [7]. Also, more shear thinning implies a higher degree of long chain branching [8].

3- Specify whether the molecular weight distribution (MWD) is narrow or broad. Higher shear thinning implies broader MWD, which in turn implies more elasticity, and this in turn implies longer stress relaxation.

4- Identifies transition regions such as $\mathrm{T}_{\mathrm{g}}, \mathrm{T}_{\mathrm{m}}$, and recognize the secondary transitions that are beyond the resolution of $\mathrm{DSC}$ [9]. $\mathrm{T}_{\mathrm{g}}$ is a reversible change of the polymer between rubbery and glassy states, and can be measured accurately by DMTA. In fact, DMTA is considered the most sensitive method for measuring a material's $T_{g}$ as a sudden change in the elastic modulus and an attendant peak in the $\tan \delta$ curve[10]. DMTA, also, tells much more about the material before and after the $\mathrm{T}_{\mathrm{g}}$.

5- Determine the degree of entanglement density (N) using equation 2 :

$$
N=\frac{G^{\prime}}{6 R T}[11]
$$

Where $G^{\prime}$ is the elastic or storage modulus, $R$ is the universal gas constant and $\mathrm{T}$, is the absolute temperature.

6- Differentiate between cross-linked and an uncrosslinked polymers: The $G$ " is much higher than the $G^{\prime}$ for the uncross-linked polymer solutions and is mainly attributed to the higher viscous property as compared to elastic property over the entire frequency range. While the $\mathrm{G}^{\prime}$ is higher than the $G^{\prime \prime}$ for the partially cross-linked polymer solutions. For hydrogel which are highly cross-linked polymer networks, both $G^{\prime}$ and $G^{\prime \prime}$ are very high and are nearly parallel to each other[12].

7- Measure the rubbery plateau modulus which is much more sensitive than is $\mathrm{T}_{\mathrm{g}}$ for detecting, for example [10].

\section{Experimental Part}

\subsection{Materials}

Carboxymethyl Cellulose (CMC) and corn starch were obtained from HIMMEDIA Laboratories Pvt. Ltd. Company (India) . Aluminum Sulfate Octadecahydrate cross-linker was obtained from REACHIM Company (USA). Nano graphite oxide was obtained from Alibaba company (China).

\subsection{Preparation of the Two Hydrogels}

To prepare the cross-linked $\mathrm{CMC} /$ starch hydrogel, an appropriate amount of starch dissolved in distilled water (DW) using water bath $80^{\circ} \mathrm{C}$ for 45 minute and added to $\mathrm{CMC}$ aqueous solution. Then, the new mixture agitated for an hour at $70^{\circ} \mathrm{C}$. The result paste was dried overnight at $100^{\circ} \mathrm{C}$ and crushed. To prepare the SHNCs hydrogel, a solution of nano GO/DW was mixed with sonication for 10 minutes and added to the $\mathrm{CMC} /$ starch mixture $(0.3 \mathrm{wt} \%)$ and allowed to mix for addition $30 \mathrm{~min}$.

\subsection{Tests}

\subsubsection{Dynamic Mechanical Thermal Analysis (DMTA)Test}

DMTA of the powdery sample (mesh 35-100) was performed using a DMTA-Triton, model (Tritec 2000DMA), England. The experiments were carried out in the temperature range of $20-300^{\circ} \mathrm{C}$, heating rate of $5^{\circ} \mathrm{C} / \mathrm{min}$ and frequency of $1 \mathrm{~Hz}$ in bending mode ,with $1 \% \mathrm{~mm}$ displacement.

\subsubsection{Rheology Test}

The rheological measurements of the water-swollen gels (1.00 g sample in $5.0 \mathrm{~mL}$ distilled water) were performed using a Paar-Physica Oscillatory Rheometer (Modulur Compact Rheometer, MCR300, Germany) with parallel plate geometry (plate diameter of $25 \mathrm{~mm}$, gap of $3 \mathrm{~mm}$ ) at $25^{\circ} \mathrm{C}$.

\section{Results and Discussions}

\subsection{DMTA Results}

Figure 1 shows a temperature sweep test on $\mathrm{CMC} /$ starch blend without cross-linker. With the temperature increase, a rapid decrease in complex modulus $\left(\mathrm{G}^{*}\right)$ occurs and the $\tan \delta$ curve goes to its maximum; the polymer is in the state of glassy transition.

The Brownian motion of an individual chain is largely inhibited by the other molecules surrounding it. When the temperature increased, the Brownian motion of the chains augment and the free volume around the polymer chains increases, which means easier flow.

Near $T_{g}$, the polymer viscoelastic properties change very quickly both with time as well as with the changing temperature.

Peaks in $\tan \delta$ and in $\mathrm{G}^{*}$ can be associated with the $\mathrm{T}_{\mathrm{g}}$, which corresponds to the ability of chains to move past each other.Therefore, based on the $\tan \delta$ pattern, the uncrosslinking $\mathrm{CMC} /$ starch blend shows two $\left(\mathrm{T}_{\mathrm{g}} \mathrm{s}\right)$ at 234.6 and at 
$265.38^{\circ} \mathrm{C}$ respectively, which indicates that this blend without cross-linker is a partially miscible blend .

Secondary transitions $(\beta)$ observed as well in the range between $61.53{ }^{\circ} \mathrm{C}$ to $80.77{ }^{\circ} \mathrm{C}$ due to the local motion of the polymer chains as opposed to large scale co-operative motion that accompanies the $\mathrm{T}_{\mathrm{g}}$.

As shown in Figure 2, cross-linking process increases the blend miscibility and converted the blend from partially miscible ( has two $\mathrm{T}_{\mathrm{g}}{ }^{\mathrm{s}} \mathrm{s} ; 234.6^{\circ} \mathrm{C}$ and $265.38^{\circ} \mathrm{C}$ ) to miscible blend with one $\mathrm{T}_{\mathrm{g}}$, which is $248^{\circ} \mathrm{C}$. This gel displays a glassy state at room temperature.

Incorporation of $0.3 \mathrm{wt} \% \mathrm{GO}$ in the cross-linked $\mathrm{CMC} /$ starch blend; in SHNCs (Figure 3) increases $\mathrm{T}_{\mathrm{g}}$ from $248^{\circ} \mathrm{C}$ to $273^{\circ} \mathrm{C}$ ). The reason can be attributed to higher stiffness of GO in comparison with the neat blend. GO, also has many functional groups which having the tendency for inter- and intra-molecular interactions which restrict chain movements.

Tan $\delta$ value before $\mathrm{T}_{\mathrm{g}}$ is considerably higher in uncross linked CMC / starch blend than in cross-linked blend. This indicates that the energy dissipation ability is decreased with incorporation of the cross-linker to the gel structure. The chain movements of uncross-linked CMC /starch blend are significantly easier than those of cross-linked blend. The same conclusion can be observed for the addition of GO sheets, which can be attributed to the fact that the additional interactions in the SHNCs limit the energy dissipation ability.

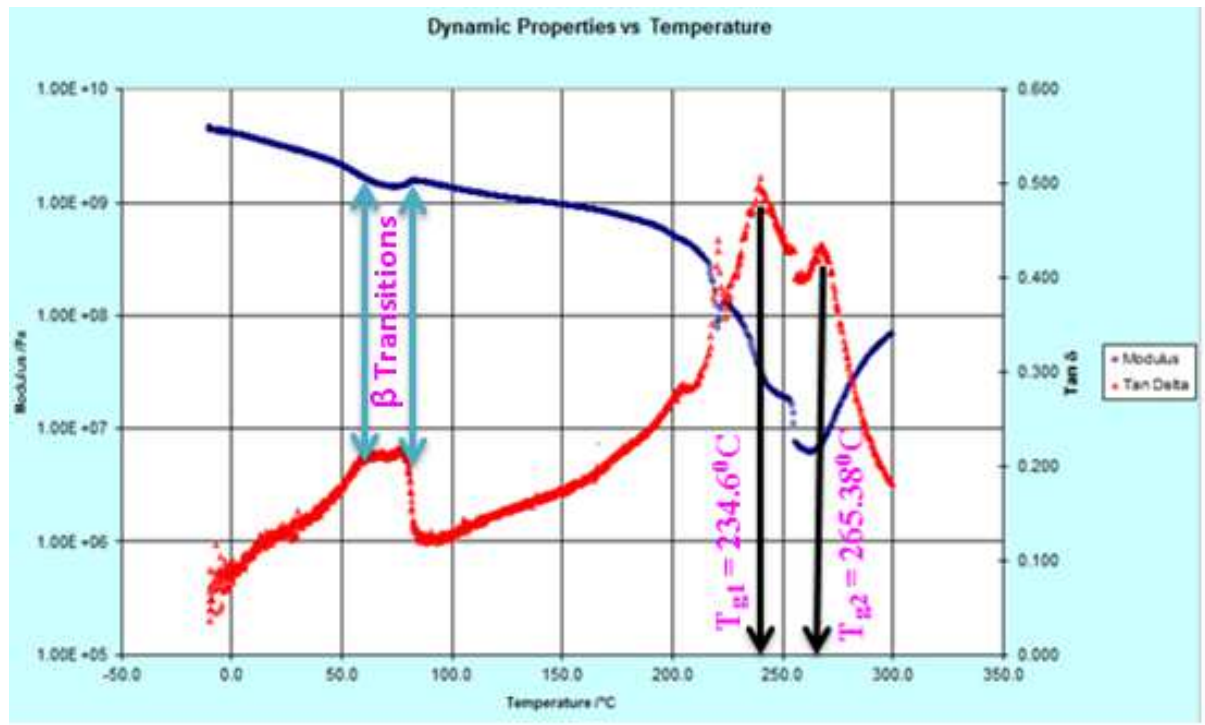

Fig (1). Complex Modulus $\left(G^{*}\right)$ and Damping factor (tan $\delta$ ) for the CMC/starch blend without cross-linker versus temperature $\left({ }^{\circ} C\right.$ )

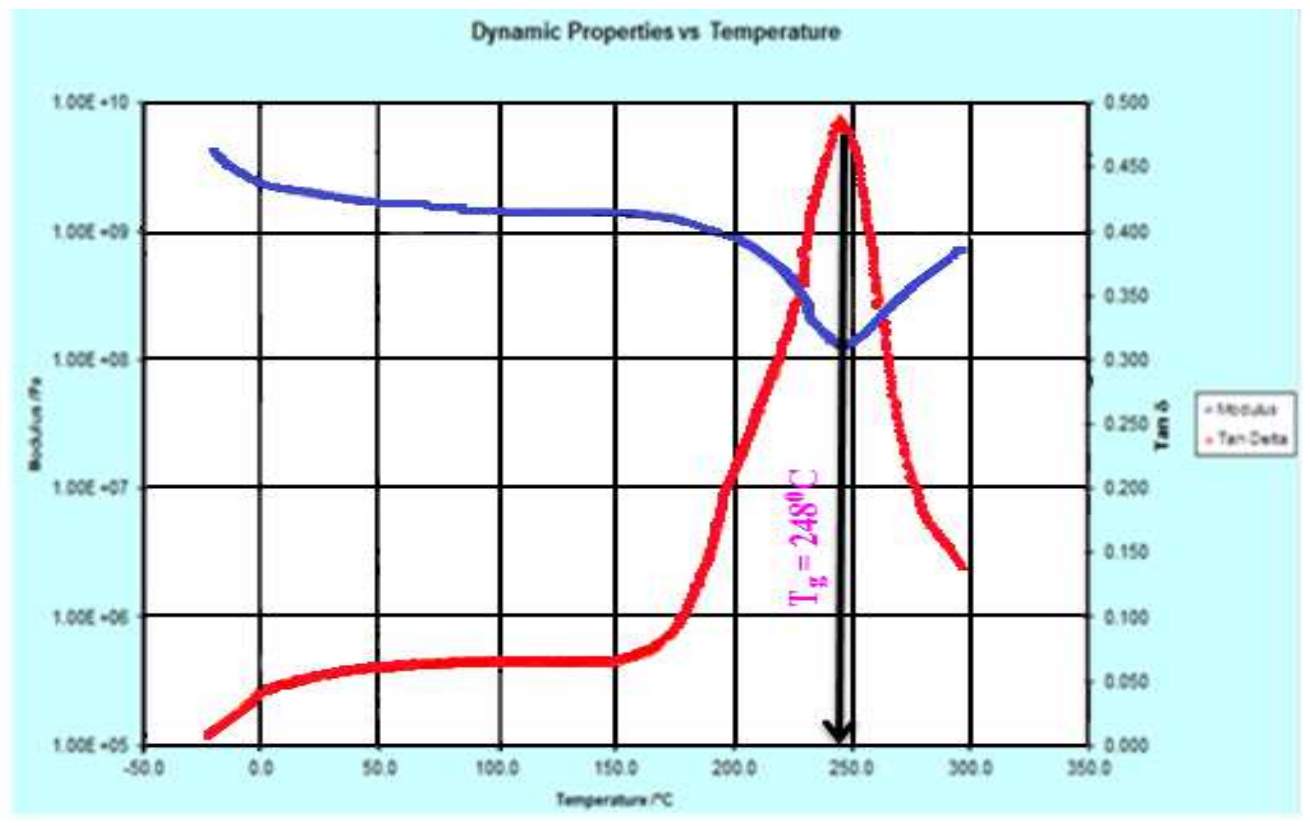

Fig (2). $G^{*}$ and tan $\delta$ for the cross-linked CMC/starch hydrogel versus temperature $\left({ }^{\circ} \mathrm{C}\right)$ 
Dynamic Properties vs Temperature

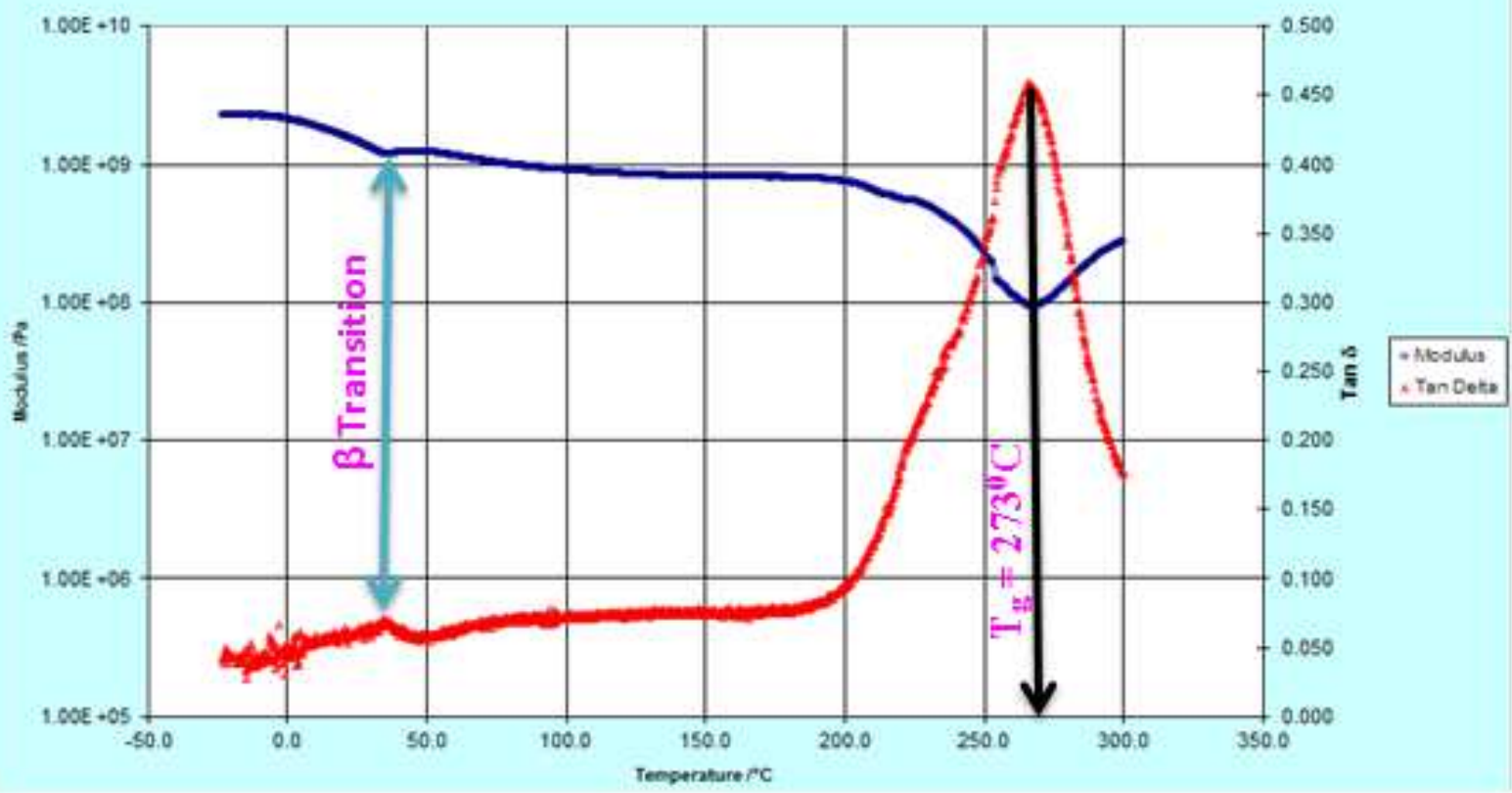

Fig (3). $G^{*}$ and tan $\delta$ for the SHNCs hydrogel versus temperature $\left({ }^{\circ} \mathrm{C}\right)$

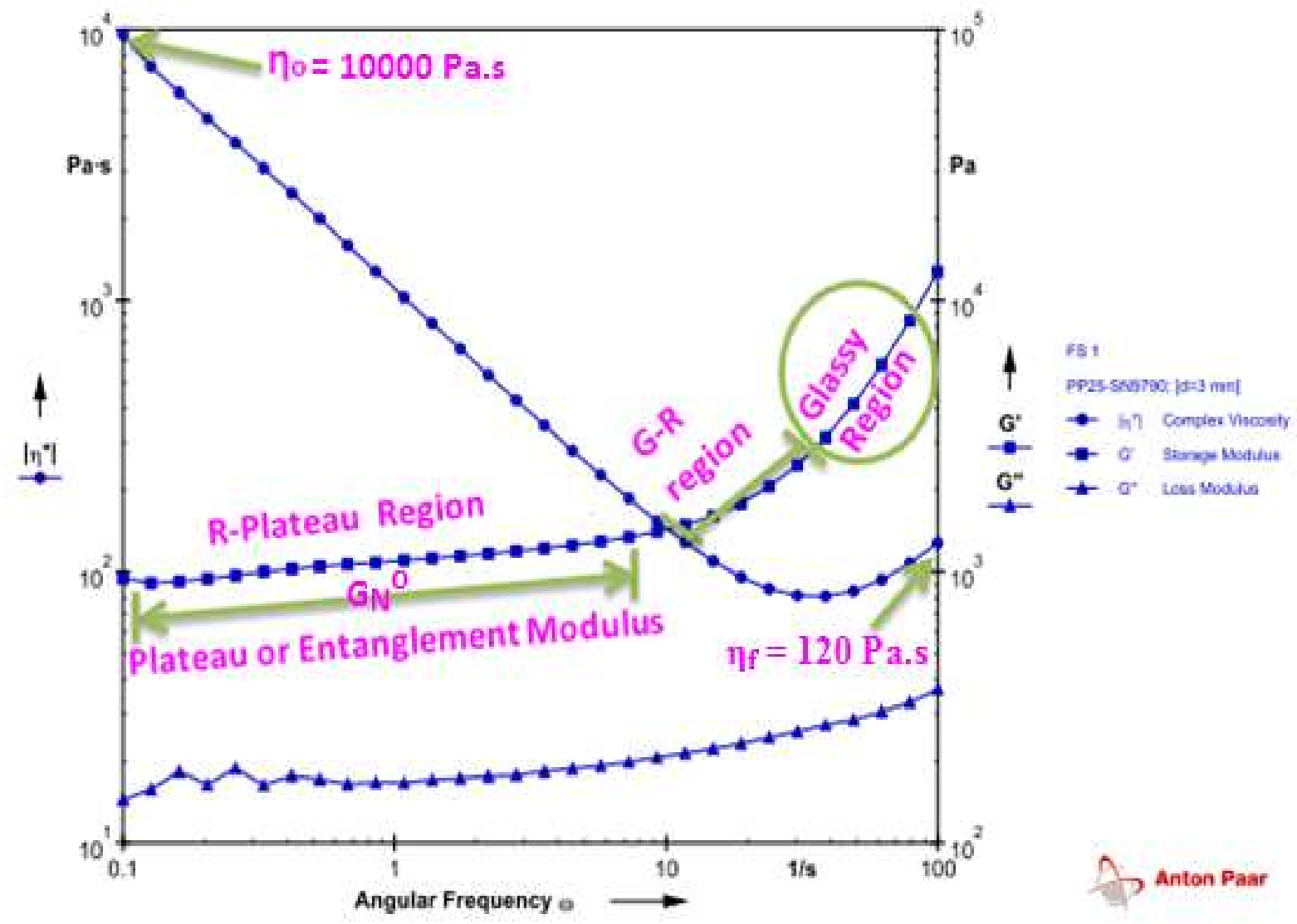

Fig (4). $G^{\prime}, G^{\prime \prime}$ and $\eta^{*}$ for the cross-linked $C M C /$ starch hydrogel versus angular frequency 


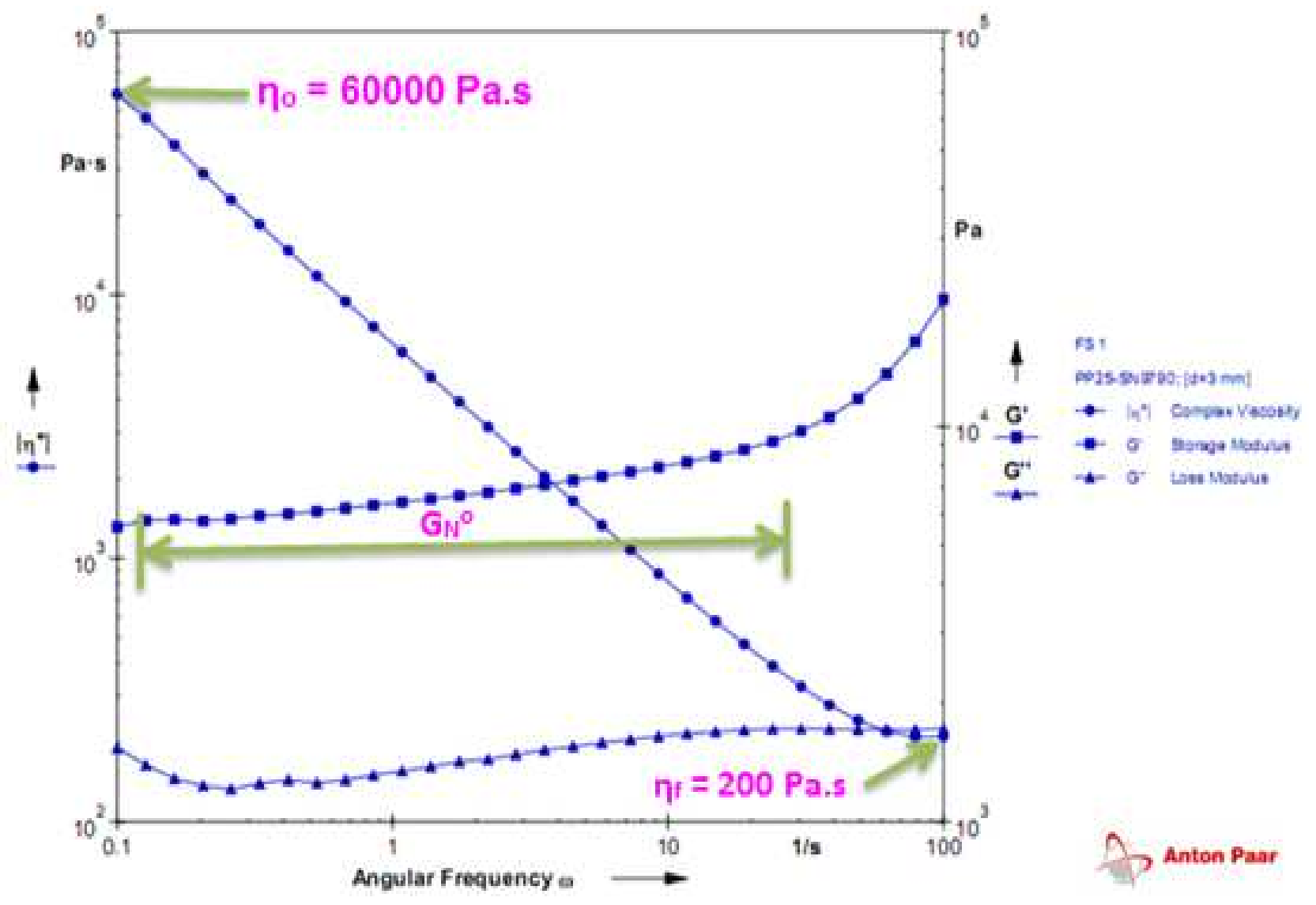

Fig (5). $G^{\prime}, G^{\prime \prime}$ and $\eta^{*}$ for the SHNCs hydrogel versus angular frequency

Above the glass-rubber transition temperature; $\mathrm{T}_{\mathrm{g}}$, large parts of the chain are free to move; their thermal energy is high enough to overcome the interaction forces, and the free volume increases with increasing temperature. The polymer is , however, not yet in the liquid condition; the coiled chains are mutually entangled. Though the chain entanglements are not permanent, because they are being disrupted with increasing temperature and also with increased time of loading, they act as temporary, physical cross-links[12].

\subsection{Rheology Results}

Figure 4 shows $G^{\prime}, G^{\prime \prime}$ and $\eta^{*}$ for the cross-linked CMC/starch blend. The plateau modulus (entanglement modulus $; \mathrm{G}_{\mathrm{N}}{ }^{\mathrm{o}}$ ) extends over angular frequency up to about 10 $\mathrm{s}^{-1}$, then the blend exhibits glass to rubber transition, and finally shows glassy state at higher frequencies[2].

The complex viscosity $\eta^{*}$ decreased from the zero-shear viscosity $\left(\eta_{\mathrm{o}}=10000 \mathrm{~Pa}\right.$.s $)$ at low frequency $\left(\omega=0.1 \mathrm{~s}^{-1}\right)$ to 70 Pa.s at intermediate frequency $\left(\omega=35 \mathrm{~s}^{-1}\right)$ and then increased to $\eta_{\mathrm{f}}=120 \mathrm{~Pa} . \mathrm{s}$ at higher frequency $\left(\omega=100 \mathrm{~s}^{-1}\right)$. Initially (at low frequencies), the chains may be highly tangled and intertwined (highly mutual wrapping of polymer chains around each other), thus the Brownian motion is insufficient to overcome the entanglement forces between the polymer chains, and with increasing the frequencies up to $35 \mathrm{~s}^{-1}$, motion increased and the chain begin to untangle and straighten( became uncoiled, more alignment and more apart from others); slips easily, therefore, the viscosity decreased.
At higher frequencies, chains haven't enough time to relax, thus became more disentangle; viscosity decreased[2]. Generally, Figure 4 shows the strong effect of temperature upon the viscosity (decreased from 10000 to 120 Pa.s), which can be attributed to the molecular structure. Usually, complexes structures such as $\mathrm{NaCMC}$ exhibit strong affect by temperature due to its rings, pendant groups, and branches.

It is clear from figure 4 that $G^{\prime}$ is higher than the $G^{\prime \prime}$ and both $\mathrm{G}^{\prime}$ and $\mathrm{G}^{\prime \prime}$ are very high and are nearly parallel to each other, which indicates that this hydrogel is highly crosslinked .Both $G^{\prime}$ and $G^{\prime \prime}$ tended to increase at higher frequencies, but the increment in $\mathrm{G}^{\prime}$ is higher than in $\mathrm{G}^{\prime \prime}$, which means that the hydrogel tend to be more elastic.

The addition of $0.3 \mathrm{wt} \% \mathrm{GO}$ sheets causes the viscosity to increase in all frequencies(Figure 5 and Table 1), which indicates the high interaction between GO sheets and the neat blend components, therefore, the entanglement modulus $\left(\mathrm{G}_{\mathrm{N}}{ }^{\circ}\right)$ extends over larger angular frequencies range; up to about 12 $\mathrm{s}^{-1}$ (in the expense of the glass to rubber region).

In view point the molecular weight, $\mathrm{GO}$ addition increases the molecular weight average since there is a linear relation between the intrinsic viscosity and the molar mass(M) according to the Mark-Houwink relation:

$$
[\eta]=k \cdot \mathbf{M}^{a}[13]
$$

in which $k$ and $a$ are constants for a given combination of polymer, solvent and temperature[13].

GO addition, also, increases the zero-shear viscosity $\left(\eta_{\mathrm{o}}\right)$ 
six times due to the increment in the intermolecular interactions[5], and disappeared the variations in $\mathrm{G}^{\prime \prime}$ at lower frequencies ; make the damping behavior more stable.

Since that the degree of entanglement density(N) can be calculated from storage modulus data (equation 2), it can be concluded from Table 1 that the value of the entanglement density(N) for SHNCs hydrogel is 4.99 times higher than its value for the same blend but without $\mathrm{GO}$, which can be attributed to the contributing of the functional groups (those available on the GO sheets surfaces) to interface with the functional groups inherently exit in the $\mathrm{CMC} /$ starch blend, which leads to increasing the entanglement between the homopolymers(CMC, and starch).

GO sheets addition, increases the complex modulus 5.41 times because the additional chain entanglement (4.99 times) inhibits the relative chain motion. It should be noted that even though secondary intermolecular(e.g. Van der Waals) bonds are much weaker than the primary covalent ones, a significant intermolecular force result from the formation of large number of Van der Waals interchain bonds.

GO sheets leads, also to the absence of the shear thickening behavior due to the strain hardening effect because with the increasing density of nanoparticles, the SHNCs hydrogel become stiffer and more fragile.

This behavior suggests that the breaking of the nanoparticles interactions dominates the onset of shear thinning [8].For both hydrogels, since $G^{\prime}$ is higher than $G^{\prime \prime}$ in the whole frequency range of the linear viscoelastic (LVE) range, the elastic behavior of the both hydrogels predominates over its viscous behavior and the swollen samples becomes increasingly rigid. In addition, with increasing frequency, i.e. low relaxation time, the samples flexibility is diminished and the swollen samples becomes increasingly rigid[13].

Also, for both hydrogels, it can be concluded that these hydrogels exhibited broad MWD because of the gradual transition of the complex viscosity curve towards the shear thinning region[2].

Table 1. Viscoelastic and rheological properties for the hydrogels at $\omega=10 \mathrm{~s}^{-1}$

\begin{tabular}{llll}
\hline Property & Without GO & With GO & Increment \\
\hline $\mathrm{G}^{\prime}(\mathrm{Pa})$ & 1611 & 8050 & 4.99 \\
$\mathrm{G}^{\prime \prime}(\mathrm{Pa})$ & 210 & 1800 & 8.5 \\
$\mathrm{G}^{*}(\mathrm{~Pa})$ & 1821 & 9850 & 5.41 \\
$\mathrm{Tan} \delta$ & 7.67 & 4.47 & 0.58 \\
$\delta($ degree $)$ & 82.57 & 77.83 & 0.94 \\
$\eta^{*}(\mathrm{~Pa} . \mathrm{s})$ & 125 & 850 & 6.8 \\
$\eta_{\mathrm{o}}(\mathrm{Pa} . \mathrm{s})^{\mathrm{a}}$ & 10000 & 60000 & 6 \\
$\eta_{\mathrm{f}}(\mathrm{Pa} . \mathrm{s})^{\mathrm{b}}$ & 120 & 200 & 1.66 \\
$\mathrm{G}_{\mathrm{N}}{ }^{\mathrm{o}}\left(\mathrm{s}^{-1}\right)^{\mathrm{c}}$ & Extends up to $\omega$ & Extends up to & 1.2 \\
\hline
\end{tabular}

a at $\omega=0.1 \mathrm{~s}^{-1} \mathrm{~b}$ at $\omega=100 \mathrm{~s}^{-1} \mathrm{c}$ at different frequencies

\section{Conclusions}

1- Cross-linking process increases the blend miscibility and converted it from partially miscible with two $\mathrm{T}_{\mathrm{g}}$ 's to miscible blend with one $\mathrm{T}_{\mathrm{g}}$.

2- Incorporation of $\mathrm{GO}$ in the cross-linked $\mathrm{CMC} /$ starch blend; in SHNCs increases $\mathrm{T}_{\mathrm{g}}$ from $248^{\circ} \mathrm{C}$ to $273^{\circ} \mathrm{C}$.

3- Tan $\delta$ value before $\mathrm{T}_{\mathrm{g}}$ in uncross-linked $\mathrm{CMC} /$ starch blend is higher than in both the cross-linked blend or in the SHNCs.

4- GO addition causes increment in some viscoelastic parameters such as $\mathrm{G}^{*}, \mathrm{G}^{\prime}$, and $\mathrm{G}^{\prime \prime}$ (5.41, 3.99, and 8.5 times respectively), and decrement in damping parameters such as $\delta$ and $\operatorname{Tan} \delta$ and make the damping behavior more stable at lower frequencies.

5- All rheological parameters $\left(\eta^{*}, \eta_{\mathrm{o}}, \eta_{\mathrm{f}}\right.$, and $\left.\mathrm{G}_{\mathrm{N}}{ }^{\mathrm{o}}\right)$ increased due to the GO addition( $6.8,6,1.66$, and 1.2 times respectively).

6- $\mathrm{G}^{\prime}$ and $\mathrm{G}^{\prime \prime}$ profiles indicate that both hydrogels are highly cross-linked.

7- GO addition leads to the absence of the shear thickening behavior and increases both the entanglement density; $\mathrm{N}$ (about five times) and the molecular weight average.

8- Both hydrogels exhibited broad MWD and in the whole frequency of the LVE range, the elastic behavior predominates over the viscous behavior and the swollen hydrogels becomes rigid.

9- With increasing frequency, hydrogels flexibility is diminished and the swollen hydrogels becomes increasingly rigid.

\section{References}

[1] J. Guan, F. Vollrath, D. Porter "Silk Quality Revealed Using Dynamic Mechanical Thermal Analysis (DMTA)" BISERICA 2013 6th BACSA International Conference: Building value Chains in Sericulture, 2013.

[2] J. Aho" Rheological Characterization of Polymer Melts in Shear and Extension: Measurement Reliability and Data for Practical Processing" Ph. D thesis, Tampere University of Technology, 2011.

[3] "Dynamic Mechanical Analysis (DMA)" G.E. Plastic Applied technology Notes,No.2.

[4] S. B. DRISCOL "Using Rheological Measurements for Quality Assurance" Quality Assurance of Polymeric Materials/Products,83-102, ASTM STP846.

[5] C. Clasen, W. Kulicke" Determination of viscoelastic and rheo-optical material functions of water-soluble cellulose derivatives" Prog. Polym. Sci. $26,2001$.

[6] G. Larson "Rheology and Structure of Complex Fluids" Oxford University Press, 1999.

[7] M. Kontopoulou" Applied Polymer Rheology: Polymeric Fluids with Industrial Applications" John Wiley \& Sons, Inc., 2012.

[8] www.pcoe.org.nz, Plastic Centre of Excellence. The University of Auckland, 2012.

[9] Thomas A. Luckenbach "DMTA: Dynamic Mechanical Thermal Analysis" Rheometrics, Inc., U.S.A. 
[10] C.Komalan, K.E. George, P.A.S. Kumar, K. T. Varughese, S. Thomas "Dynamic mechanical analysis of binary and ternary polymer blends based on nylon copolymer/EPDM rubber and EPM grafted maleic anhydride compatibilizer" eXPRESS polymer letters vol.1, No.10 (2007) 641-653.

[11] K.PAL, A.K. BANTHI, D.K.MAJUMDAR" Polymeric Hydrogels: Characterization and Applications - A mini review" Designed Monomers and Polymers, 12,2009.
[12] A.K Van der Vegt "From Polymer to Plastics" Delft University Press, The Netherlands, 2002.

[13] M.J Ramazani-Harandi, M.J Zohuriaan-Mehr, A .A. Yousefi, A, Ershad-Langroudi, K. Kabiri " Test Method: Rheological determination of the swollen gel strength of superabsorbent polymer hydrogels" Polymer Testing 25,470-474, 2006. 